\title{
Análisis del modelo motivacional de las ocho anclas de Edgar Schein y el modelo motivacional empleado por una empresa del sector financiero en la ciudad de Cartagena
}

\author{
Analysis of the motivational model of Edgar Schein's eight anchors and the \\ motivational model used by a company in the financial sector in the city of Cartagena \\ Rosario Cuadrado-Álvarez* \\ Universidad Libre - Colombia \\ ORCID iD: https://orcid.org/0000-0003-0406-8750 \\ rosario.cuadrado@unilibre.edu.co \\ Luis Miguel Pineda-Portacio \\ Universidad Libre - Colombia \\ ORCID iD: https://orcid.org/0000-0002-2160-1652 \\ luism-pinedap@unilibre.edu.co \\ Yeferson Castellanos-Oviedo \\ Fecha de recepción: 22/07/2020 \\ Fecha de evaluación: 13/08/2020 \\ Fecha de aceptación: 20/10/2020 \\ Cómo citar: Cuadrado-Álvarez, R., Pineda-Portacio, L., \\ \& Castellanos-Oviedo, Y. (2020). Análisis del modelo mo- \\ tivacional de las ocho anclas de Edgar Schein y el mode- \\ lo motivacional empleado por una empresa del sector \\ fnanciero en la ciudad de Cartagena. Revista Cientifica An- \\ fibios, 3(2), 54-65. https://doi.org/10.37979/afb.2020v3n2.73
}

Universidad Libre - Colombia

ORCID iD: https://orcid.org/0000-0002-4657-4240

yeffersone-castellanoso@unilibre.edu.co

\section{Leydis Santana-Díaz}

Universidad Libre - Colombia

ORCID iD: https://orcid.org/0000-0002-2949-7888

leydisp-santanad@unilibre.edu.co

* Autor a quien debe ser dirigida la correspondencia

\section{Resumen}

La motivación en el trabajo ha sido a la larga de la historia empresarial objeto de estudio, dado que ella le aporta los estímulos requeridos a una persona para que se conecte con el puesto y la organización. El plan motivacional de una organización debe responder a las necesidades reales de las personas que la conforman, por ello se conceptualiza y se identifican las ventajas de la teoría de las Ocho Anclas aplicables en la organización.

Palabras clave:

Motivación; organización; teorías motivacionales; colaboradores; modelo motivacional.

\begin{abstract}
Motivation at work has been the object of study throughout history, given that she provides the required stimuli to a person to connect with the position and the organization. The motivational plan of an organization must respond to the real needs of the people who make it up, for this reason, the advantages of the theory of the Eight Anchors applicable in the organization are conceptualized and identified.
\end{abstract}

Keywords:

Motivation; organization; motivational theories; collaborators; motivational model. 
Análisis del modelo motivacional de las ocho anclas de Edgar Schein y el modelo motivacional empleado por una empresa del sector financiero en la ciudad de Cartagena

\section{Introducción}

La motivación en el trabajo puede concebirse como un proceso mediante el cual un colaborador impulsado por fuerzas internas o que actúan sobre él, inicia, dirige y mantiene una conducta orientada a alcanzar determinados estímulos que le permiten la satisfacción de sus necesidades, mientras simultáneamente intenta alcanzar las metas de la organización. Es por ello, que ha sido objeto de estudio a lo largo de la historia empresarial, con el propósito de entender los intereses de las personas en cuanto al desarrollo de sus actividades dentro de la empresa y al poder precisar los aspectos subjetivos que pueden afectar la dimensión del trabajo.

La investigación surge del entendimiento que deben tener las organizaciones sobre la motivación y las acciones que pueden emprender para que sus colaboradores se sientan motivados; lo cual implica conocer las necesidades e intereses de las personas en diferentes escenarios organizacionales o en condiciones laborales específicas, evidenciando la importancia de hacer seguimiento a los niveles de satisfacción del colaborador como herramienta que contribuye al alcance de los objetivos organizacionales.

La empresa objeto de estudio, pertenece al sector financiero, en ella se comparará el modelo motivacional implementado en la organización con el propuesto por Dr. Edgar Schein en la Teoría de las Ocho Anclas, identificando las ventajas y desventajas en cada caso.

\section{Motivación en el trabajo}

La palabra motivación es el resultado de la combinación de los vocablos latinos motus (traducido como "movido") y motio (que significa "movimiento") (Definicion, 2008a). A juzgar por el sentido que se le atribuye al concepto desde el campo de la psicología y de la filosofía, una motivación se basa en aquellas cosas que impulsan a un individuo a llevar a cabo ciertas acciones y a mantener firme su conducta hasta lograr cumplir todos los objetivos planteados (Herrera, s.f). La noción, además, está asociada a la voluntad y al interés; en otras palabras, puede definirse la mo- tivación como la voluntad que estimula a hacer un esfuerzo con el propósito de alcanzar ciertas metas (Definicion, 2008b).

En los años 30 un experimento realizado en una planta en los suburbios de Chicago, reveló que la calidad de las relaciones del individuo con sus pares (compañeros de trabajo) y su jefe inmediato, influían de manera directa en su productividad, más que cualquier otra variable física del entorno laboral.

Luego, durante las décadas de los 40 y 50 se pensó que en la medida que el colaborador veía satisfecha sus necesidades básicas, éste se motivaba en su trabajo y eso lo hacía más productivo. En los años 60 se identificó que ciertas necesidades no materiales motivaban a los colaboradores, otros materiales generaban insatisfacción; lo cual sirvió de base para establecer que el diseño integral de los puestos de trabajos podría tener colaboradores motivados.

A partir de la segunda década del siglo $\mathrm{XX}$, se produjo un cambio del conductismo al neoconductismo, aparece así, el concepto de impulso (Actualidad empresa, 2013). En este periodo predomina una visión del sujeto pasiva, donde no se tiene en cuenta el consciente sino el inconsciente, por lo que el hombre no tiene capacidad de dirigir sus conductas

Desde el punto de vista organizacional la motivación proviene del elemento humano, es decir, de personas capacitadas para el desempeño de cargos y que cumplen un papel fundamental tanto en la toma de decisiones como el proceso operativo y además influyen directamente con la consecución de los objetivos trazados que permiten lograr la competitividad del mercado de bienes y servicios. En la actualidad, encontramos organizaciones que generan sus propias políticas motivacionales, sin considerar lo expuesto por autores reconocidos y enfocadas en los incentivos monetarios, excluyendo otros factores motivacionales relevantes para los colaboradores.

\section{Aporte de autores a su conceptualización}

Teniendo en cuenta el objetivo principal de la investigación y con el propósito de sopor- 
tar esta exploración la cual busca compilar un análisis donde se caracterice la motivación de la empresa objeto de estudio, como recurso primordial para lograr un ambiente laboral armonioso, colaboradores comprometidos, procesos con mínimo de errores, logro de objetivos empresariales, ganancias a los inversionistas y clientes satisfechos; se citan autores que a través de la historia y según sus proyectos/teorías han identificado factores internos y externos en los que se ven envueltos al momento de realizar las funciones asignadas desde el momento de su contratación, estos autores han definido la motivación como:

Jerarquía de las Necesidades de Maslow: $\mathrm{Su}$ autor clasifican las necesidades de acuerdo con la hipótesis de que dentro de todo ser humano existe una jerarquía de las siguientes cinco necesidades (Maslow, 1954), a saber:

- Fisiológicas: incluye el hambre, la sed, el abrigo, el sexo y otras necesidades corporales

- De seguridad: incluye la seguridad y protección de daños físicos y emocionales.

- Sociales: incluye afectos, la sensación de pertenencia, aceptación y amistad.

- De Ego: incluye factores internos de estima, como el respeto a uno mismo, la autonomía y los logros; y factores externos como el status, el reconocimiento y la atención.

- De autorrealización: el impulso de ser lo que se es capaz de ser; incluye el crecimiento, alcanzar el potencial de uno y la autosatisfacción.

Teoría de los factores de Herzberg: Esta teoría revela que "una vez que las personas han logrado satisfacer sus niveles básicos de necesidades, desplazan sus requerimientos hacia otra dimensión, hacia otros factores motivacionales inherentes a la persona." (Herzberg, 1966). Según el autor existen dos tipos de factores que intervienen en las relaciones laborales:

Motivadores: son los orientados a garantizar la satisfacción de las personas tanto en el desempeño de sus labores como en sus propias expectativas, y abarcan aspectos como Logros, reconocimiento, promoción, retos, asignación de responsabilidades.

Higiénicos: tienen que ver tanto con el contexto donde se desempeña el trabajo como con el trato que las personas reciben: Ambiente laboral, recursos materiales, beneficios sociales, sueldos, relaciones personales, políticas institucionales

Las diferencias entre los factores básicos y los motivacionales, reside en que lo primeros no proporcionan una satisfacción adecuada a los intereses de las personas, sino antes bien, solamente lograr "aliviar el estado de insatisfacción", liberando al individuo para que se motive más si su trabajo es suficientemente rico en oportunidades de desarrollo (Sosa, 2013).

\section{Teoría De Las Necesidades De Mcclelland}

Propone que las personas poseen unas necesidades específicas adquiridas y moldeadas a lo largo de la vida. David McClelland, expuso en "The Achieving Society" (1961) su Teoría de las Necesidades y realizó sus investigaciones con directivos y gerentes exitosos de empresas de países industrializados. Efectuó comparaciones con ejecutivos y gerentes de empresas de países poco desarrollados.

Mcclelland en sus estudios utilizó la técnica proyectiva denominada Test de Apercepción Temática (TAT); teniendo en cuenta los resultados obtenidos, intentó explicar cómo las necesidades: Logro o Realización, Poder y Afiliación afectan las acciones de las personas, a saber:

Necesidades de Logro: Las personas cuyo impulsor motivador es esta necesidad, realizan, establecen y logran metas desafiantes, asumen riesgos para lograr sus objetivos y buscan recibir retroalimentación sobre sus progreso, logros o desempeño; no obstante, suelen trabajar solos.

Necesidad de Poder: Las personas que poseen el impulsor motivador de poder se caracterizan por querer contralar e influir en los 
demás; disfrutan la competitividad, el ganar con argumentos, del estatus y el reconocimiento.

Necesidad de Afiliación: Las personas que poseen el impulsor motivador de poder disfrutan de la compañía de otros, el pertenecer a un grupo y tener a cargo a otros. Son personas queridas, crean un buen ambiente laboral y la colaboración está por encima de la competencia.

Teoría De Las Inteligencias Múltiples De Howard Gardner: En 1984 Gardner publicó su libro Frames of Mind: The Theory of Multiple Intelligences, en donde hablaba de la teoría de las múltiples inteligencias, hasta entonces se consideraba una persona inteligente aquella a la que se le daban muy bien las matemáticas o las letras, siendo los deportistas quienes se llevaban la peor parte de este reparto de cociente intelectual.

El autor en su teoría reconoce ocho habilidades mentales, aunque no descarta la posibilidad que sean más, a saber:

- Inteligencia lingüística: Es la inteligencia más valorada porque nos ayuda a ser hábiles con las palabras, a utilizarlas de forma más adecuada, aprender idiomas o expresarnos mejor. Hace referencia a lo que hablamos, escribimos y lo que comprendemos de lo expresado por otras personas.

- Inteligencia lógico-matemática: Forma parte del núcleo de las pruebas de inteligencia; permite resolver con habilidad los problemas algorítmicos, memorizar números o datos, comprender la lógica de las cosas o detectar una falacia lógica.

- Inteligencia musical: Permite apreciar, distinguir y elaborar los ritmos, las melodías, las diferentes estructuras musicales, el timbre, el tono o los instrumentos que participan en una pieza musical.

- Inteligencia espacial: Es la inteligencia relacionada con la habilidad de crear espacios en nuestra imaginación y manipularlos mentalmente; la podemos utilizar para resolver rompecabezas, conducir autos o hallar la salida en un laberinto.

- Inteligencia cinético-corporal: Le permite a la persona conectarse fácilmente con su cuerpo, tener un mejor control y expresarte a través de los movimientos. Ejemplo los bailarines, actores y deportistas.

- Inteligencia interpersonal: Permite comprender a los demás y su comportamiento, tiene que ver con la empatía. Es un tipo de inteligencia esencial en las relaciones con otros, es utilizada para negociaciones, en lo comercial.

- Inteligencia intrapersonal: Tiene que ver con el modo en que aprendemos a analizar todo aquello que ocurre en nuestra mente, a comprendernos mejor a nosotros mismo.

- Inteligencia naturalista: Habilidad relacionada con la improvisación, se utiliza para entornos creativos y novedosos.

Teoría De Las Ocho Anclas De Edgard Schein: Así como somos distintos física y emocionalmente, también lo somos en lo que nos motiva; para Schein existen ocho anclas que las personas buscan en su vida.

El planteamiento anterior, conduce a aceptar como válida, la conclusión formulada por E. Schein, en su obra Psicología de la Organización, cuando plantea: "el principal motivador de la persona adulta es la necesidad de mantener y desarrollar un concepto de sí mismos y un concepto bueno de sí mismo" (Schein, 1980).

\section{Las ocho anclas definidas por Schein}

- Seguridad: Se refiere a la búsqueda de seguridad, dicha y estabilidad. Podría ser un empleo sin riesgos o un trabajo seguro.

- Creatividad: Hace énfasis en la necesidad de innovar y crear cosas nuevas. Artistas, diseñadores o profesionales en mercadeo, son carreras que pueden interesar a quienes tienen esta ancla. 
- Autonomía e independencia: Se refiera a la necesidad de tener libertad de movimiento a la hora de desarrollar su carrera profesional, tener su propio estilo y actuar según su voluntad. Escritores, asesores, consultores o empresarios.

- Dirección: Esta ancla es sobre quienes buscan dirigir y coordinar para lograr cosas que están fuera de su alcance.

- Técnico / Funcional: Se refiere a las personas que se centran en un campo del conocimiento de forma específica. Les interesa formarse en la empresa y sentirse indispensables.

- Desafío: Personas interesadas en retos, desafíos y oponentes que proporcionen desafíos o competencias. Están en la búsqueda de empresas que les ofrezcan retos o desafíos interesantes.

- Servicio o dedicación: Personas con un compromiso muy profundo que expresan a través de su labor profesional, por ejemplo, médicos, bomberos o guardabosques.

- Estilo de vida: Personas que eligen un estilo de vida y lo anteponen a todo. Encontrar una organización donde pueda llegar luego de dejar a los hijos en el colegio.

\section{Metodología}

El estudio realizado es de carácter descriptivo, dado que busca identificar los aspectos motivacionales de los colaboradores en una empresa del sector financiero de la ciudad de Cartagena, haciendo un comparativo entre la Teoría Motivacional de las Ocho Anclas del autor Edgard Schein y el plan motivacional aplicado por la empresa objeto de estudio.

En cuanto a la técnica de recolección y procesamiento de la información es por fuente primaria, mediante un instrumento cerrado desarrollado y patentado por el autor Edgar Schein; el cual será aplicado a la totalidad de la población de la empresa objeto de estudio.

\section{Beneficios de la teoría motivacional de las ocho anclas}

Las organizaciones que ponen de relieve las necesidades de más alto nivel ayudan a crear buscadores de motivación y al mismo tiempo proporcionan oportunidades para el comportamiento motivado de los individuos que están desenvolviéndose en esos niveles altos.

En concordancia con lo hasta aquí planteado, se analizarán dentro del área crítica Motivación, los siguientes parámetros:

- Realización personal.

- Reconocimiento del aporte.

- Responsabilidad.

- Adecuación de las condiciones de trabajo.

En la valoración de la Realización personal se considera la realización integral de la persona, que oportunidades de autorrealización tendría en un contexto organizacional, donde ponga en práctica sus habilidades y destrezas.

Cuando las satisfacciones en el desempeño del trabajo superan las expectativas de los colaboradores, éstos se convierten en aliados (se comprometen con la organización); ahora bien, los niveles de satisfacción pueden decrecer cuando no se hace un Reconocimiento del Aporte, por parte de la administración. Cuando la organización reconoce y da crédito al esfuerzo realizado por cada persona o grupo en la ejecución de las tareas asignadas para el logro de los objetivos institucionales, posee un alto potencial motivador porque satisface las necesidades de realce del ego.

Para facilitar la satisfacción de estas necesidades, es necesario que cada colaborador esté consciente de su importancia dentro del ambiente organizacional, por la valoración de sus tareas como imprescindibles para alcanzar las metas de la institución y tengan el deseo sincero de cooperar para ello con los demás.

La Responsabilidad, en una organización se mide por el grado de compromiso con que 
los colaboradores deciden asumir, consciente y voluntariamente, sus deberes en respuesta a las demandas de trabajo, manifestando la motivación existente. Para ello debe contar con la confianza por parte de los jefes, lo cual es un factor que incrementa la motivación personal.

Finalmente, las condiciones ambientales físicas y psico-sociales en que se realiza el trabajo, así como la calidad y cantidad de los recursos que se suministran para el cumplimiento de las funciones asignadas, deben ser congruentes con la naturaleza misma del trabajo (Sosa, 2013).

Cuando la adecuación de las condiciones de trabajo no se presenta, se produce insatisfacción en el colaborador, repercutiendo en bajos niveles de desempeño y en la calidad de los productos o servicios producidos. Cabe mencionar, que las adecuadas condiciones ambientales, pueden ser un factor desencadenante de insatisfacción, también pueden considerarse una fuente significativas de satisfacción y motivación.

\section{Caracterización de la empresa}

La organización financiera objeto de estudio lleva pocos años en el mercado, pero en el corto tiempo ha logrado captar una importante cifra de colocación y captación de productos. Esta entidad hace parte de una cooperativa que nació en la ciudad de Cali y actualmente es la segunda más grande de Latinoamérica.

El direccionamiento estratégico es su clave del éxito, es muy particular y eficiente porque es deber de cada uno de los colaboradores conocer la misión, visión, valores corporativos, política integral de sistema de gestión y los objetivos de calidad con esto garantizan que todos sus colaboradores hablen el idioma de la empresa y lo trasmitan a los clientes bajo los criterios establecidos.

El proceso de selección de los colaboradores para ingresas a la empresa es a través de su matriz principal, en la cual se ejecutan todo el proceso de pruebas y entrevistas con los postulantes al perfil solicitado, una vez seleccionado el candidato es enviado a la regional más cercana para su proceso de capacitación que dura alrededor de dos semanas y una vez finalizado es llevado a la oficina donde cumplirá con las asignaciones operativas y comerciales, junto a su nuevo grupo de trabajo.

\section{Caracterización de los sujetos participantes}

Este es un grupo heterogéneo de personas con diferentes creencias, edades, género, antigüedad en la empresa, puesto que desempeñan; a continuación, se podrán observar el detallado de estos aspectos:

Tabla 1. Categorización de los sujetos participantes.

\begin{tabular}{|c|c|c|c|c|c|}
\hline Cargo & Edad & Genero & Antigüedad & Área & Nivel de escolaridad \\
\hline Gerente & 53 & Femenino & Más de 20añ & Comercial & Especialista \\
\hline Director de Servicio & 38 & Femenino & 10 años & $\begin{array}{l}\text { Comercia/ } \\
\text { operativo }\end{array}$ & Profesional \\
\hline Asesor de Ventas & 26 & Femenino & 2 años & Comercial & $\begin{array}{c}\text { Estudiante de carrera } \\
\text { Profesional }\end{array}$ \\
\hline Asesor de ventas & 27 & Femenino & 2 años & Comercial & Profesional \\
\hline Asesor de Servicios & 25 & Masculino & 1 año & $\begin{array}{l}\text { Comercia/ } \\
\text { operativo }\end{array}$ & Profesional \\
\hline Asesor de Servicios & 33 & Masculino & 8 años & $\begin{array}{l}\text { Comercia/ } \\
\text { operativo }\end{array}$ & Técnico \\
\hline Asesor de Servicios & 30 & Masculino & 5 años & $\begin{array}{l}\text { Comercia/ } \\
\text { operativo }\end{array}$ & Tecnólogo \\
\hline Analista de Cartera & 42 & Femenino & 12 años & Comercial & Profesional \\
\hline
\end{tabular}

Fuente: Elaboración propia 


\section{Características del plan motivacional de la empresa objeto de estudio}

El plan motivacional que rige a la empresa y que es socializado con sus colaboradores por diferentes medios, es el siguiente:

- Bonificación anual por cumplimento de la empresa, se recibe una vez al año.

- Auxilio de alimentación anual (este auxilio es descontado de la liquidación si el contrato se finaliza antes del año.

- Bonificación por cumplimiento de metas mensuales.

- Auxilio de transporte anual (este auxilio es descontado de la liquidación si el contrato se finaliza antes del año).

- Créditos por libranza a baja tasa de interés.

- Capacitación permanente de los productos bancarios.

- Fiesta de fin de año.

\section{- Ascensos o promoción de Cargos}

Las ventajas del plan motivacional es que la empresa ofrece compensaciones extralegales diferentes a las establecidas por la Ley, a través de esta los colaboradores pueden satisfacer alguno de sus intereses y cumplir con los objetivos establecidos por la organización.

La desventaja de este plan motivacional puede ser el concentrar la motivación en lo económico, el $80 \%$ del plan está enfocada la compensación monetaria, dejando de lado otros intereses que los colaboradores quieran tener, como por ejemplo, flexibilidad en el horario, seguimiento a su desempeño para el cumplimiento de los objetivos mensuales, extender los beneficios al núcleo familiar, incentivar el desarrollo personal y laboral de sus colaboradores.

Características motivacionales según la teoría de las ocho anclas
Para determinar las características del ancla de carrera los encuestados, en términos generales, identifican que es importante para ellos y qué buscan en su carrera profesional, en otras palabras, cuál es la posible orientación de sus trayectorias profesionales. El encuestado no se limita a responder en términos del pasado. $\mathrm{Su}$ ancla de carrera es algo más complejo que un mero reflejo de experiencias pasadas; incluye también una autoevaluación de sus habilidades y capacidades, con una clara proyección hacia el futuro.

El Cuestionario consta de 41 preguntas y afirmaciones donde se debe responder que tan de acuerdo o en desacuerdo se está con esa afirmación, dándole un puntaje que va del 1 al 10. Al finalizar de responder se sumarán los resultados obtenidos en cada variable para saber cuál o cuáles son sus anclas más predominantes, para así lograr entender cuáles son sus prioridades personales y dentro de la organización.

Para el análisis de la información recopilada se realizará de manera individual el estado actual de cada colaborador, identificando sus anclas de carrera para conocer qué tan conectado están los intereses particulares con los intereses de la organización, en este punto se resaltarán las 3 anclas de carrera más fuertes en cada colaborador, también de analizarán los resultados de manera grupal para identificar anclas de carreras en común las cuales darán a conocer cuáles son los aspectos en común por los cuales los colaboradores son contratados y así tener una certeza de que la selección de personal está o no está alejada a los perfiles profesionales buscados por la organización y por ultimo establecer un diagnóstico de los métodos motivacionales usados actualmente por la empresa y los métodos motivacionales que se podrían emplear al usar el modelo de las 8 anclas de carrera de Edgar Schein.

\section{Resultados consolidados y análisis grupal}

A continuación, se representa el número de anclas de carrera con mayores coincidencias obtenidas por los colaboradores de la oficina 
Análisis del modelo motivacional de las ocho anclas de Edgar Schein y el modelo motivacional empleado por una empresa del sector financiero en la ciudad de Cartagena

objeto de estudio; se puede observar que cuatro de los ocho colaboradores su ancla fuerte es Servicio/Dedicación, seguida de Emprendedor con incidencia tres colaboradores de ocho. Se podría decir que la pasión por el servicio, por cumplir la asignación de las metas, por cumplir sus intereses particulares, también podrían incluirse irse de viaje, iniciar un negocio propio, comprar un carro, adquirir vivienda o sobresalir del resto del grupo; a saber:

Tabla 2. Ancla predominante en la muestra según el número total de encuestados

\section{No. De anclas predominantes en los colaboradores}

\section{TEMA}

$\begin{array}{cc}1 & \text { Servicio/Dedicación } \\ 2 & \text { Emprendedor }\end{array}$

3

4

5

6

7

8

9

\section{ANCLA}

Reto

Seguridad/Trabajo estable seguridad/Estabilidad Geográfica

Técnica/Funcional

Autonomía/Independencia

Estilo de vida
Directiva
PUNTUACIÓN TOTAL

RANKING

1
2
3
4
4
4
4
4
4

Fuente: Elaboración propia

A continuación, se detalla el resultado de la suma de los puntajes individuales de cada uno de los colaboradores y sus respectivas anclas multiplicados por $100 \mathrm{y}$ divididos por el puntaje total que se podía obtener.

Tabla 3. Ancla Predominante según la puntuación general

\begin{tabular}{ccccc}
\hline \multicolumn{2}{c}{ Resultado grupal oficina } \\
TEMA & ANCLA & $\begin{array}{c}\text { PUNTUACIÓN } \\
\text { TOTAL }\end{array}$ & $\%$ & RANKING \\
1 & Técnica/Funcional & 250 & $62.5 \%$ & 8 \\
2 & Directiva & 294 & $73.5 \%$ & 7 \\
3 & Autonomía/Independencia & 299 & $74.7 \%$ & 6 \\
4 & Seguridad/Trabajo estable & 186 & $77.5 \%$ & 5 \\
5 & seguridad/Estabilidad Geográfica & 120 & $50 \%$ & 9 \\
6 & Servicio/Dedicación & 350 & $87.5 \%$ & 2 \\
7 & Reto & 312 & $78 \%$ & 4 \\
8 & Estilo de vida & 319 & $79.7 \%$ & 3 \\
9 & Emprendedor & 353 & $88.2 \%$ & 1 \\
\hline
\end{tabular}

Fuente: Elaboración propia

Se aprecia la variación del ancla predominante con respecto al cuadro anterior, da lugar a Emprendedor como la real ancla con mayor incidencia dentro del grupo de colaboradores de la oficina según sus resultados individuales, dejando en un segundo lugar a Servicio y entrando al ranking Estilo de Vida como tercera ancla dominante en el grupo.

Estos resultados permiten identificar las necesidades reales que tienen los colabora- dores de la empresa objeto de estudio y por la cual deberían enfocar los métodos motivacionales, para lograr que sean efectivos y se logren contrarrestar o mitigar las falencias que actualmente son evidencia de un buen modelo motivacional estructurado.

Teniendo en cuenta los resultados grupales se puede inferir que el plan de compensación motivacional no está alineado con los intereses de los colaborados pues el ancla emprendedor 
que el colaborador tenga derecho a ser reconocido por su esfuerzo laboral, que se le proporcionen las herramientas necesarias para llevar a cabo ideas innovadoras que irían en pro de la organización, tener flexibilidad a la hora de tomar algunas decisiones y facilitarle los medios para que lleven a cabo su plan de vida individual; al enfocar los esfuerzos del plan motivacional de la empresa hacia lo que los colaboradores requieren para estar motivados seria benefactor tanto para los clientes, socios, dueños de la empresa y sus colaboradores.

Identificar las oportunidades de mejora del modelo motivacional empleado por la empresa teniendo en cuenta las características de la teoría de las ocho anclas

El Plan motivacional propuesto, basándose en los resultados de la aplicación de la encuesta de la teoría de las 8 anclas de Edgard Schein, es el siguiente:

- Auxilio óptico (Estilo de Vida)

- Auxilio de incapacidad médica (Estilo de Vida)

- Medicina prepagada (Seguridad y Estilo de Vida)

- Crédito de vivienda (Emprendedor)

- Auxilio de estudio para empleados e hijos (Emprendedor y Servicio)

- Becas por promedio a mejores estudiantes (Emprendedor)

- Primas extralegales en junio y diciembre (Emprendedor)

- Créditos de fomento microempresarial (Emprendedor)

- Convenios de fomento al deporte (Estilo de Vida)

- Carrer Break (suspensión del contrato por 3 meses sin remuneración para que el empleado viaje al exterior a estudiar otro idioma) garantizándole la continuación del contrato al regreso (Servicio)
Cerca de la familia (salir una hora antes los días de los cumpleaños de los familiares) para comprarles torta, regalo o simplemente estar el día especial con ellos)

- Torneos entre empleados

- Día de la familia

\section{- Auxilio de boda}

Teniendo en cuenta las debilidades del plan motivacional actual de la empresa objeto de estudio y conociendo el perfil motivacional de cada uno de los colaboradores, es menester que se realicen ajustes para provocar motivación; lo ideal es que el área encargada de socializar los métodos motivacionales en la organización considere lo propuesto y tenga en cuenta no solo los factores económicos sino también de integración, salud, higiene y aspectos familiares de los colaboradores.

Además, el plan de motivación empleado actualmente está enfocado en cubrir las necesidades monetarias, movilidad y promociones; para poder acceder a estos beneficios los colaboradores deben mes a mes cumplir. Está claro que se debe premiar a los colaboradores que hacen bien su trabajo, pero sin recurrir al temor, la situación económica actual por la que está atravesando el país es desfavorable para la colocación de productos y captación de recursos y afecta a todas las entidades financieras.

La rigurosidad del seguimiento al cumplimiento de metas dejan de lado la parte emocional y motivacional de los colaboradores, en el caso de los empleados operativos además de tener que estar concentrados en el área de caja recibiendo efectivo y a la vez colocando productos es una línea delgada en donde se debe atender bien y rápido al cliente, no descuadrar la operación y a la vez colocar un producto nuevo al cliente, esto sin contar el tiempo de espera de los clientes próximos a ser atendidos sin que se enojen con el asesor de servicio e impongan una queja en contra del servicio de la oficina. No siendo suficiente para lograr las metas, en ocasiones deben sacar de su espacio de almuerzo para gestionar telefónicamente entrega de tarjetas crédito y uso de créditos rota- 
tivos; lo que resulta desgastante y no motivante hacia la tarea por cumplir.

El modelo de las Ocho Anclas, considera el estímulo o interés de cada colaborador, obteniendo con ello no solo un mayor y mejor rendimiento en las actividades y tareas a desarrollar, sino que se mantendrán vínculos de largo plazo y colaboradores que se sentirán plenamente identificados con la empresa por permitirles lograr metas que, aunque no tienen relación con la empresa hacen parte de los objetivos personales de los colaborados.

Entre los diferentes aspectos y las diferentes herramientas motivacionales que se deberían incluir están los aspectos sociales, todos los colaboradores tienen momentos especiales y significativos con sus seres más allegados (cumpleaños, grados, matrimonio, calamidades domesticas), es responsabilidad de la organización permitirle vivir estos momentos que marcan un punto clave en sus vidas y estos automáticamente serán recompensados por el colaborador.

El grupo de colaboradores destaca como su ancla predominante la de emprendedor, es por esto que la organización deberá proporcionar las herramientas para que sus colaboradores tomen esa iniciativa, empezando desde la capacitación (estudios formales) hasta el fomento de las ideas propias de cada uno (créditos de fomento empresarial),Para su segunda y tercer ancla que son Servicio y estilo de vida es importante ayudarles a construir un espacio con seres allegados por esto el aspecto de vivienda ayudara a motivar esa ancla.

A continuación, se presenta un cuadro de resumen en donde se ven los aspectos a considerar según la teoría de las ocho anclas basado en las personalidades de los colabores y si actualmente son incluyente según el plan motivacional de la empresa objeto de estudio

Tabla 4. Comparativo de compensaciones motivacionales entre la empresa objeto de estudio y la teoría de las ocho anclas

\begin{tabular}{ccc}
\hline Aspectos & $\begin{array}{c}\text { Plan Motivacional Actual Empresa } \\
\text { Objeto de Estudio }\end{array}$ & $\begin{array}{c}\text { Plan Motivacional Teoría 8 } \\
\text { anclas }\end{array}$ \\
\hline Familiares & Excluyente & Incluyente \\
Sociales (permiso especial) & Excluyente & Incluyente \\
Emprendimiento & Excluyente & Incluyente \\
Superación personal & Incluyente & Incluyente \\
Capacitación & Incluyente & Incluyente \\
Vivienda & Excluyente & Incluyente \\
Reconocimientos & Incluyente & Incluyente \\
Monetario & Incluyente & Incluyente \\
Alimentación y transporte & Incluyente & Excluyente \\
\hline
\end{tabular}

Fuente: Elaboración propia

\section{Conclusión}

Teniendo en cuenta los resultados obtenidos, se puede concluir que una organización puede efectuar su plan de motivación de manera propia sin guiarse de un autor o estudio de motivación, pero que es menester no descuidar el talento humano y sus motivos/intereses y que estos a su vez estén acorde a sus necesidades reales para no arriesgar los recursos invertidos y que se garantice la efectividad de estos, estos incentivos pueden ser socializados mensual, bimestral, semestral y anualmente; lo impor- tante es tener en cuenta los aspectos integrales del colaborador para mantenerlo motivado en el trabajo.

Es importante resaltar que la empresa objeto de estudio con su plan motivacional ha logrado escalar muchos peldaños en tan poco tiempo, lo cual es bueno pero si quieren lograr metas más ambiciosas deberán tener en cuenta la caracterización de sus colaboradores que le permitirá conocer de manera real las necesidades de sus colaboradores y poder desarrollar un plan motivacional estructurado y eficaz den- 
tro de las posibilidades empresariales.

La aplicabilidad del modelo motivacional de la teoría de las ocho anclas de Edgard Schein ayuda al área de talento humano a conocer plenamente las necesidades de cada uno de sus colaboradores, lo cual tendrá un impacto positivo que permitirá tomar medidas favorables de acuerdo intereses de la empresa y escoger a los colabores más aptos para realizar diferentes labores. Entre los beneficios más palpables al contar con un personal motivado están: Una mejor imagen de la empresa, mayor compromiso con la empresa, mayor rendimiento laboral, Más y mejores ideas de mejora, Mayor competitividad empresarial y Menos fugas de cerebros. Este último es de suma importancia pues ha sido un factor problema muy enmarcado dentro de la organización que fue objeto de estudio.

Se le recomienda al área de gestión humana, enfocar las directrices y analizar el plan motivacional actual de la organización, hacia el fortalecimiento de unas buenas relaciones Jefe-Colaborador, establecer metas claras y razonables de acuerdo a la situación actual del país, ofrecer buenas compensaciones, ser im- parcial al momento de promover al personal y buscar siempre medios adicionales de motivación.

Independientemente de su tamaño, toda empresa está en condiciones de mantener altos niveles de motivación para esto y gracias a la encuesta se conocen las personalidades y expectativas en cuanto a intereses personales y laborales de cada uno de los colaboradores por tal motivo se deberá alinear el perfil del colaborador con las especificaciones del cargo con el propósito de disminuir o eliminar las frustraciones o desmotivaciones a causa de realizar un trabajo paralelo a su perfil.

Colaboradores motivados mejor niveles de desempeño, porque se sienten bien en su trabajo y lo hacen con una mayor eficacia. Esto hace que se consiga un mayor rendimiento laboral y mayores beneficios para la empresa además si se sienten valorados y motivados son capaces de tener nuevas ideas y transmitirlas a sus superiores. Puede que algunas no sean válidas o aplicables, pero se pueden conseguir ideas interesantes para mejorar o rendir más. También son más creativos y hacen mejor su trabajo.

\section{Referencias}

Actualidad empresa (2013). Teorías de la motivación su evolución y clasificación parte-1. http://actualidadempresa.com/teorias-de-la-motivacion-su-evolucion-y-clasificacion-parte-1/

Definicion (2008a). Definición de motivación. https://bit.ly/3gcbdtr

Definicion (2008b). Definición de motivación. https://definicion.de/motivacion/

García, R. (2011). Estudio sobre la motivación y los problemas de convivencia escolar. Trabajo fin de Máster). España: Universidad de Almería. Recuperado de repositorio. ual. http://repositorio. ual.es/bitstream/handle/10835/1179/Garcia_Esquivel_Raquel.pdf

Gardner, H. (1984). The Theory of Multiple Intelligences: Frames of Mind. London, UK: Heimann.

Herrera, K (s.f) Un emprendedor motivado es el principio de grandes cosas. https://bit.ly/2E97PB9

Herzberg, F. I. (1966). Work and the Nature of Man.

Maslow, A. H. (1954). Personality and motivation. Harlow, England: Longman, 1, 987.

McClelland, D. C. (1961). The achieving society. Princeton, NJ: D. Van Norstrand Company. Inc., 1961.

Schein, E. (1980). H., Psicología de la organización, Motivación y supuestos sobre la naturaleza humana, Punto 5. 
Análisis del modelo motivacional de las ocho anclas de Edgar Schein y el modelo motivacional empleado por una empresa del sector financiero en la ciudad de Cartagena

Sosa, A. (2013). Clima organizacional. Consultado 27 de marzo de 2017. Recuperado de: http://plusformacion.com/Recursos/r/clima-organizacional 